\title{
ON QUADRATIC FUNCTIONALS
}

\section{Peter Šemrl}

In this note a general solution of the problem of the characterisation of quadratic functionals posed by Vukman is given.

Theorem. Let $A$ be a complex *-algebra with identity $e$ and let $X$ be a vector space which is also a unitary left $A$-module. Suppose there exists a mapping $Q: X \longrightarrow$ $A$ with the properties

(i) $Q(x+y)+Q(x-y)=2 Q(x)+2 Q(y)$ for all pairs $x, y \in X$, and

(ii) $Q(a x)=a Q(x) a^{*}$ for all $x \in X$ and all $a \in A$.

Under these conditions the mapping $B(\cdot, \cdot): X \times X \rightarrow A$ defined by the relation

$$
B(x, y)=(1 / 4)(Q(x+y)-Q(x-y))+(i / 4)(Q(x+i y)-Q(x-i y))
$$

satisfies the following:

$$
\begin{aligned}
& B(\cdot, \cdot) \text { is additive in both arguments; } \\
& B(a x, y)=a B(x, y) \\
& B(x, a y)=B(x, y) a^{*}, \text { for all pairs } x, y \in X \text { and all } a \in A \\
& Q(x)=B(x, x) \text { for all } x \in X
\end{aligned}
$$

Remark: A functional $Q: X \longrightarrow A$ which satisfies (i) and (ii) is called an $A$ quadratic functional and a mapping $B: X \times X \longrightarrow A$ for which conditions (1) and (2) are fulfilled is called an $A$-sesquilinear functional. If $A$ is the complex number field then this result reduces to Kurepa's extension of the Jordan-Neumann theorem which characterises pre-Hilbert space among all normed spaces.([3])

Proof: As in the proof of Kurepa's result (see [3], [5] and also [6]) one can prove that the function $W(\cdot, \cdot)$ defined by relation $W(x, y)=Q(x+y)-Q(x-y)$ is additive in both variables. Therefore the same is true for the functional $B$. A short computation shows that $Q(x)=B(x, x)$ for all $x \in X$. Hence it remains to prove (2). For this purpose we define a new functional $S: A \times A \longrightarrow A$ by $S(a, b)=a B(x, y) b^{*}-B(a x, b y)$

Received 17 March 1987

This work was supported by the Research Council of Slovenia 
where $x$ and $y$ are fixed vectors. From the fact that $B$ is biadditive it follows that the functional $S$ is also biadditive. Using (ii) one can easily obtain

$$
S(c a, c b)=c S(a, b) c^{*}, \quad a, b, c \in A
$$

A short computation yields $S(i a, b)=i S(a, b)$ and $S(a, i b)=-i S(a, b)$. For any four elements $a, b, c, d \in A$ we have that $S(a b, a c)+S(a b, d c)+S(d b, a c)+S(d b, d c)$ $=S((a+d) b,(a+d) c)=(a+d) S(b, c)\left(a^{*}+d^{*}\right)=a S(b, c) a^{*}+d S(b, c) a^{*}+a S(b, c) d^{*}$ $+d S(b, c) d^{*}$. This yields $S(a b, d c)+S(d b, a c)=d S(b, c) a^{*}+a S(b, c) d^{*}$. Replacing $d$ and $c$ by $e$ we get

$$
S(a b, e)+S(b, a)=S(b, e) a^{*}+a S(b, e)
$$

Let us put the element $i a$ instead of $a$. We obtain

$$
i S(a b, e)-i S(b, a)=-i S(b, e) a^{*}+i a S(b, e)
$$

Comparing (4) and (5) we see that $S(a b, e)=a S(b, e)$ and $S(b, a)=S(b, e) a^{*}$. Replacing $b$ by $e$ by using the relation $S(e, e)=0$ we complete the proof.

This result was proved in [4] and [8] under the stronger assumption that $A$ is a Banach *-algebra (see also [6] and [7]) using the fact that such algebras have enough invertible elements. It should be mentioned that in the proof of the present general result an idea similar to those of Davison [1] was used.

\section{REFERENCES}

[1] T.M.K. Davison, 'Jordan derivations and quasi-bilinear forms', Comm. Algebra 121 (1984), 23-32.

[2] S. Kurepa, 'The Cauchy functional equation and scalar product in vector spaces', Glasnik Math. Fiz.-Astr. 19 (1964), 23-36.

[3] S. Kurepa, 'Quadratic and sesquilinear functionals', Glas. Mat. Fiz.-Astr. 20 (1965), 79-92.

[4] P. Śemrl, 'On quadratic and sesquilinear functionals', Aequationes Math. 31 (1986), 184-190.

[5] P. Vrbová, 'Quadratic and bilinear forms', Časopis Pĕst. Mat. 98 (1973), 159-161.

[G] J. Vukman, 'A result concerning additive functions in hermitian Banach *-algebras and an application', Proc. Amer. Math. Soc. 91 (1984), 367-372.

[7] J. Vukman, 'Some results concerning the Cauchy functional equation in certain Banach algebras', Bull. Austral. Math. Soc. 31 (1985), 137-144.

[8] J. Vukman, 'Some functional equations in Banach algebras and an application', Proc. Amer. Math. Soc. (to appear).

Institute of Mathematics, Physics and Mechanics

University of Ljubljana

P.O. Box 543

61001 Ljubljana

Yugoslavia 